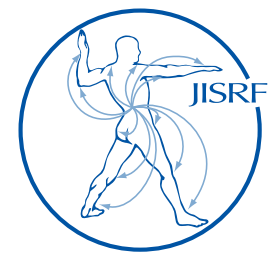

\title{
The History, Technical Specifications and Efficacy of Plasma Spray Coatings Applied to Joint Replacement Prostheses
}

\author{
McCabe A ${ }^{1}$, Pickford $M^{2}$, Shawcross $J^{1}$
}

\begin{abstract}
Thermal plasma sprayed coatings are designed to improve both the biocompatibility and durability of implantable medical devices, and include pure titanium, cobalt/chrome alloy and hydroxyapatite. Coated joint replacements have now been in continuous clinical use for thirty years and are applied to products manufactured or used in Europe, North America, South America, Africa, Asia and Australasia. Prostheses incorporating such coatings have been successfully implanted into several million of patients worldwide and to date there have been very few reports of any failure of an implant which could be attributed to problems with, or failure of, the coating. This paper summarises the early history of cementless prostheses and subsequent development, specification, validation, regulatory requirements and clinical performance of thermal plasma spray coatings provided by Accentus Medical.
\end{abstract}

Keywords: plasma; spray; coatings; joint; replacement; prostheses

Level of Evidence: AAOS Therapeutic Level V

\section{Introduction and Background}

During the development of total hip replacement, during the 1950s and 1960s, the early prostheses such as the Charnley Hip were incorporated into the bone using an acrylic cement. This was made from polymethyl methacrylate, which had originally been developed for use in the dental industry, a couple of decades before. Such a method of fixation has proved to be very successful and the technique is still in use today, especially for older, low demand patients. However, long-term follow up of patient cohorts, showed that cement was susceptible to fatigue failure in high demand patients and that the resultant cement debris led to osteolysis and death of the host bone around the implant. This phenomenon became known as cement disease
1 Andrew McCabe BSc PhD, James Shawcross BSc PhD Accentus Medical Ltd, 528.10 Unit 2, Rutherford Avenue, Harwell Oxford, Didcot, OX11 0DF, UK

(Direct reprint requests to Andrew McCabe)

2 Martin Pickford BSc PhD

Craneswater Consulting Ltd, 37 Downland Close, Locks Heath, Southampton, SO31 6WB, UK

(C) 2016 McCabe, Pickford, Shawcross. All rights reserved. Authors retain copyright and grant the journal right of first publication with the work. Reconstructive Review follows the Creative Commons Attribution-NonCommercial CC BY-NC. This license

allows anyone to download works, build upon the material, and share them with others for non-commercial purposes as long as they credit the senior author, Reconstructive Review, and the Joint Implant Surgery \& Research Foundation (JISRF). An example credit would be: "Courtesy of (senior author's name), Reconstructive Review, JISRF, Chagrin Falls, Ohio". 
[1], a problem that was widely researched and published in the 1980s.

There was clearly a need to eliminate cement from the artificial joints of young, active or obese patients if successful outcomes were to be achieved in these patient groups.

As a direct result of this, the 1980s saw the development of a large range of hips, knees and shoulders that were designed to be used without cement, in a press-fit fashion. Many of these designs such as the Harris Galante (Zimmer), PCA (Howmedica) and Taperloc (Biomet) incorporated porous metal coatings into the surface of the prosthesis, aimed at promoting a mechanical interlock between the bone and the prosthesis during the healing process. These surfaces were variously composed of a "spaghetti" like wire construct [2] attached to the prosthesis using a technique called diffusion bonding, sintered metal beads [ [3] applied by a similar method, or by a porous layer of metal powder [4] applied by a thermal plasma spray method. After some early failures due to poor mechanical design, such techniques became very popular for younger patients. The National Joint Registry of England, Wales and Northern Ireland reports that over half of total joint replacements are performed today in a cementless fashion $[\underline{5}, \underline{6}]$, with good long-term outcomes. Although there are still many different types of porous metal coatings and surface modifications on the market, plasma spray coatings have become by far the most popular. Firstly, it is a very cost effective solution that is readily applicable to all designs of joint replacement. Secondly, the results are good even at long-term follow up $[4,7,7, \underline{8}, \underline{2}]$.

In 1984, another important technology was introduced to the market. An English surgeon named Ronald Furlong introduced a cementless hip stem incorporating a surface coating of a ceramic called hydroxyapatite, which was also applied using a thermal plasma spraying technology. Hydroxyapatite is a constituent of natural bone and the coating was designed to promote the growth of new host bone around the prosthesis during the healing process.

The results of the Furlong HAC stem have been excellent [7], and many of today's world leading brands of cementless hips and knees incorporate a coating of hydroxyapatite or a bi-coating of plasma spray metal (to create a roughened surface) followed by a sequential layer of hydroxyapatite.

\section{Purpose of Coatings and Types Available}

Plasma spay coatings on joint replacement prostheses are designed to encourage new bone formation around an implant, thereby improving fixation and long-term survi- vorship of the artificial joint. Many studies have shown that a well applied coating does produce the required outcome $[4,7,8,2]$, especially in total hip and knee replacement.

Accentus Medical has developed a global reputation for the development and proprietary production of its Acusure ${ }^{\circledast}$ range of high quality plasma spray coatings aimed at meeting the requirements of its customers around the world.

The quality and validation of such coatings are governed by a series of international standards (e.g. ISO, ASTM) devised and controlled by regulatory authorities around the world, (e.g. EU Notified Bodies, FDA). These requirements are described in the following sections of this paper.

\section{International Standards and Regulatory Requirements}

The plasma spraying of metal and hydroxyapatite coatings for orthopaedic implants is governed by a number of ISO and ASTM standards and FDA good practice guidelines. These cover the input materials specification, the control of the coating process, and the properties of the resultant coating.

\section{TITANIUM RAW MATERIAL \& COATING}

The specification and validation of the input raw materials used to produce orthopaedic implants, and to produce titanium coatings applied to orthopaedic implants, are covered by several key documents.

ISO 5832-2 "Implants for surgery - metallic materials - unalloyed titanium" and ASTM F67 "Specification for unalloyed titanium for surgical implant applications" specifically refer to the ingot or other feedstock from which orthopaedic implants are machined, and the material used to produce powder that is subsequently sprayed onto implants to produce a titanium coating. ASTM F1580 "Titanium Powders for Coating of Surgical Implants" specifies the physical and chemical characteristics of the powder for plasma spraying.

The specification and validation of titanium coatings applied to orthopaedic implants are covered by ISO 131791, "Implants for surgery - Plasma sprayed unalloyed titanium coatings on metallic surgical implants", and the FDA $510(\mathrm{k})$ guidelines "Guidance for Industry on the Testing of Metallic Plasma Sprayed Coatings on Orthopaedic Implants to Support Reconsideration of Post-market Surveillance Requirements", Issued February 2, 2000 [미].

The guidelines require a range of metallurgical analy- 
sis, microstructure and mechanical properties of the coated surface:

- Metallurgical analysis of the materials

- Microstructure of the modified surface

- Mechanical properties of modified surface

- Manufacturing details

These specifications are to assess the suitability of the coating for its primary purpose, to produce a rough finish to assist with bone integration onto the orthopaedic component. The properties of the coating may differ from that of the input raw material.

ASTM and ISO standards apply to the majority of the tests including:

- ASTM F-1044 "Shear testing of calcium phosphate coatings and metallic coatings"

- ASTM F-1147 "Standard test method for tension testing of calcium phosphate and metallic coatings"

- ASTM F-1160 "Shear and bending fatigue testing of calcium phosphate and metallic medical and composite calcium phosphate/metallic coatings"

- ASTM F-1854 "Stereological evaluation of porous coatings on medical implants"

- ASTM F-1978 "Measuring abrasion resistance of metallic thermal spray coatings by using the Taber ${ }^{\mathrm{TM}}$ Abraser"

- ISO 9220 "Metallic and related coatings: scanning electron microscope method for measurement of local thickness of coating by examination of cross sections".

\section{HYDROXYAPATITE RAW MATERIAL \& COATING}

The specification and validation of both the input raw materials and the hydroxyapatite coatings applied to orthopaedic implants are covered by a number of key documents, mainly BS ISO 13779-2, "Implants for Surgery - Hydroxyapatite Part 2 Coatings of hydroxyapatite" and guidelines produced by the FDA " $510(\mathrm{k})$ Information Needed for hydroxyapatite Coated Orthopaedic Implants", March 10, 1995 [11].

The FDA guidance document requires the following information for validation of the hydroxyapatite coatings:

- Particle size and particle size distribution, pore volume and porosity;

- Coating thickness and tolerance as measured by scanning electron microscopy;

- Chemical analysis of the hydroxyapatite powders before and after coating, including $\mathrm{Ca} / \mathrm{P}$ ratios, elemental analysis;

- Bond strength of the hydroxyapatite coating;

- Solubility products of hydroxyapatite before and after coating;
- Dissolution rate of hydroxyapatite before and after coating;

- XRD patterns of hydroxyapatite before and after coating;

- Infrared spectra of hydroxyapatite before and after coating;

- The coating tested shall be as close to final product to market as practically possible (so processed, cleaned, packaged and sterilised).

ASTM and ISO standards apply to the majority of the tests. For example:

- ISO 13779-2 "Hydroxyapatite - Coatings of hydroxyapatite"

- ISO 13779-3 "Hydroxyapatite - Chemical analysis and characterisation of crystallinity and phase purity"

- ISO 13779-4 "Hydroxyapatite - Determination of coating adhesion strength"

- ASTM F 1044 "Shear testing of calcium phosphate coatings and metallic coatings"

- ASTM F 1147 "Tension testing of calcium phosphate and metallic coatings"

- ASTM F 1185 "Composition of hydroxyapatite for surgical implants"

- ASTM F 2024 "X-ray diffraction determination of phase content of plasma sprayed hydroxyapatite coatings"

There is some variation worldwide, sometimes in the tests themselves, others in the number of tests required for statistical validity. The authors' experience in regulatory requirements includes Europe, North America, South America, Africa, Asia and Australasia. Dr Andrew McCabe is the convenor of the ISO TC150 ceramics working group involved with hydroxyapatite coatings of medical devices.

\section{Coatings - Specifications, Testing and Validation}

The authors have experience validating plasma sprayed titanium (chemically pure titanium, CpTi) and hydroxyapatite coatings used in the coating of orthopaedic implants.

\section{PROCESS AND SOFTWARE VALIDATION}

The equipment and the process of applying plasma spray titanium and hydroxyapatite coatings to specific customer products requires validation by Installation Qualification (IQ), Operational Qualification (OQ), Performance Qualification (PQ) and Computer Software Qualification (CQ) methodology, as recommended e.g. by the Global Harmonisation Task Force. [12] 


\section{COATING DESIGN VALIDATION}

The coatings used on an orthopaedic product are subject to design validation requirements. In addition to the design criteria for the implant itself (performed by the device manufacturer), the coating vendor produces coating design validation masterfiles to support the device manufacturer's product. It should be demonstrated that the coating does not itself cause any negative effect on the final coated product, for example shear fatigue properties.

\section{TITANIUM COATING}

Table 1 summarises the physical, mechanical and chemical testing, the international ISO and ASTM standards to which the testing is performed, the regulatory limits and the typical test results achieved with a quality titanium coating.

Table 1. Properties of plasma sprayed titanium

\begin{tabular}{|c|c|c|c|}
\hline Property & Standards & Requirement & $\begin{array}{l}\text { Typical } \\
\text { Value }(*)\end{array}$ \\
\hline $\begin{array}{l}\text { Static } \\
\text { Tensile }\end{array}$ & $\begin{array}{l}\text { ASTM F 1147, FDA } \\
\text { Guidance [10] }\end{array}$ & $>22 \mathrm{MPa}$ & $40 \mathrm{MPa}$ \\
\hline $\begin{array}{l}\text { Static } \\
\text { Shear }\end{array}$ & $\begin{array}{l}\text { ASTM F 1044, FDA } \\
\text { Guidance [10] }\end{array}$ & $>20 \mathrm{MPa}$ & $38 \mathrm{MPa}$ \\
\hline $\begin{array}{l}\text { Shear } \\
\text { Fatigue }\end{array}$ & $\begin{array}{l}\text { ASTM F 1160, FDA } \\
\text { Guidance [10] }\end{array}$ & $\begin{array}{l}10 \mathrm{MPa}, 10[7] \\
\text { cycles }\end{array}$ & PASS \\
\hline Abrasion & $\begin{array}{l}\text { ASTM F 1978, FDA } \\
\text { Guidance [10] }\end{array}$ & $\begin{array}{l}<65 \mathrm{mg} @ 100 \\
\text { cycles }\end{array}$ & PASS \\
\hline Nitrogen & \multirow{10}{*}{ ISO 13179-1 } & $<5 \%$ & $<2 \%$ \\
\hline Oxygen & & $<10 \%$ & $<4 \%$ \\
\hline Hydrogen & & $<0.2 \%$ & $<0.1 \%$ \\
\hline Carbon & & $0.08 \%$ & $0.02 \%$ \\
\hline Iron & & $0.50 \%$ & $0.04 \%$ \\
\hline Silicon & & $0.06 \%$ & $0.012 \%$ \\
\hline Chlorine & & $0.20 \%$ & $0.04 \%$ \\
\hline Sodium & & $0.50 \%$ & $0.19 \%$ \\
\hline Magnesium & & $0.50 \%$ & $<0.01 \%$ \\
\hline Titanium & & Balance & Balance \\
\hline
\end{tabular}

* These are typical results obtained for a quality titanium coating. The data significantly exceed the minimum regulatory requirements.

In addition, thickness, porosity and density measurements are performed and reported. In these cases there are no pass/fail criteria documented in the associated standards and guidelines.

A number of OEMs, and a few independent suppliers, offer titanium and hydroxyapatite coating by 'vacuum' plasma spray (VPS). This process uses a reduced pressure of inert gas (typically 0.1 atmospheres), and claims mechanical and chemical properties substantially in ex- cess of regulatory requirements, although at a premium price. Standard air plasma spray (APS) coatings are less dense than VPS, mainly due to less particle melting. They contain increased, but acceptable levels of oxygen, nitrogen and hydrogen due to pick up from the atmosphere and achieve coating strength in excess of the minimum regulatory requirements.

The Acusure ${ }^{\circledR}$ range uses a mixture of inert (nitrogen and argon) and reducing (hydrogen) gases within its air plasma to carefully control the structure and properties of the coating. The properties of such coatings are therefore more favourable than those generally found in the open literature for APS coatings.

A typical SEM cross section microstructure of an angular titanium coating, sprayed onto a titanium substrate (LHS white area), is shown in Figure 1a, with an SEM image of the surface topography in Figure 1b. There is the option of several alternative grades of angular titanium, providing coatings of different roughness.

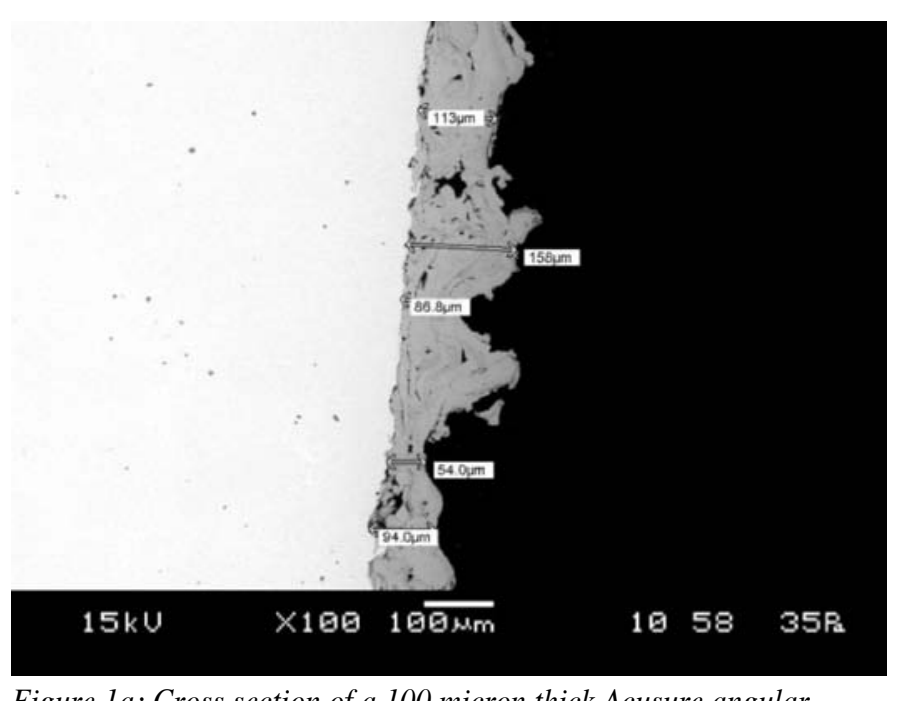

Figure 1a: Cross section of a 100 micron thick Acusure angular titanium coating.

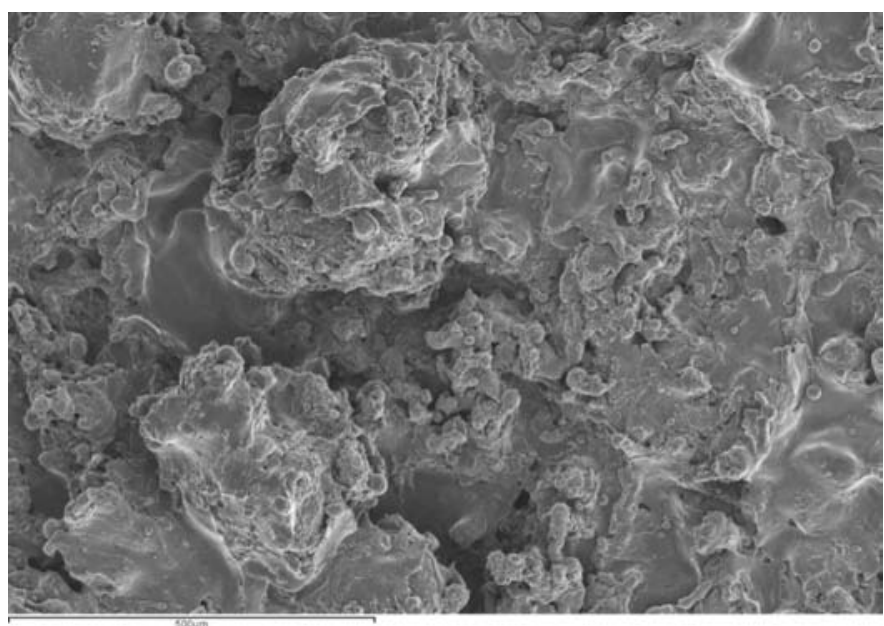

Figure 1b: The surface of an Acusure angular titanium coating. 


\section{HYDROXYAPATITE COATING}

Table 2 summarises the physical, mechanical and chemical testing, the international ISO and ASTM standards to which the testing is performed, the regulatory limits and the test results achieved with a quality hydroxyapatite coating.

In addition, thickness, porosity and density measurements, infra-red spectroscopy, and solubility and dissolution testing are performed and reported. In these cases there are no pass/fail criteria documented in the associated standards and guidelines.

The selection of hydroxyapatite raw material, and the process parameters used, result in the production of a high purity and exceptionally high crystallinity hydroxyapatite coating ( $80 \%$ crystallinity compared to a minimum regulatory requirement of 45\%). Re-testing after five-year ageing demonstrates a very stable coating with a low level of solubility and dissolution rate.

An example of SEM cross section microstructure of a hydroxyapatite coating, sprayed directly onto a grit blast-

Table 2. Properties of plasma sprayed hydroxyapatite coating

\begin{tabular}{|c|c|c|c|}
\hline Property & Standards & Requirement & $\begin{array}{l}\text { Typical } \\
\text { Value }\left(^{*}\right)\end{array}$ \\
\hline Static Tensile & $\begin{array}{l}\text { ASTM F } \\
\text { 1147, FDA } \\
\text { Guidance [11] }\end{array}$ & $>15 \mathrm{MPa}$ & $28 \mathrm{MPa}$ \\
\hline Static Shear & $\begin{array}{l}\text { ASTM F } \\
\text { 1044, FDA } \\
\text { Guidance [11] }\end{array}$ & None & $>30 \mathrm{MPa}$ \\
\hline $\begin{array}{l}\text { XRD phase } \\
\text { analysis:- }\end{array}$ & \multirow{8}{*}{$\begin{array}{l}\text { ISO 13779-2 } \\
\text { ASTM F } 2024 \\
\text { FDA } \\
\text { Guidance [11] }\end{array}$} & & \\
\hline $\mathrm{Ca} / \mathrm{P}$ ratio & & $1.65-1.76$ & 1.70 \\
\hline Crystallinity & & $45 \%$ & $80 \%$ \\
\hline$\%$ HAP & & $>50 \%$ & $92.7 \%$ \\
\hline$\% \alpha$-TCP & & $<5 \%$ & $3.2 \%$ \\
\hline$\% \beta$-ТCP & & $<5 \%$ & $4.1 \%$ \\
\hline$\%$ TCCP & & $<5 \%$ & $\begin{array}{l}\text { None } \\
\text { detected }\end{array}$ \\
\hline$\% \mathrm{CaO}$ & & $<5 \%$ & $\begin{array}{l}\text { None } \\
\text { detected }\end{array}$ \\
\hline Trace elements:- & \multirow{6}{*}{$\begin{array}{l}\text { ISO 13779-2 } \\
\text { ASTM F } \\
1185-03 \\
\text { FDA } \\
\text { Guidance [11] }\end{array}$} & & \\
\hline As & & $3 \mathrm{ppm}$ & $<3 \mathrm{ppm}$ \\
\hline $\mathrm{Cd}$ & & $5 \mathrm{ppm}$ & $<5 \mathrm{ppm}$ \\
\hline $\mathrm{Hg}$ & & $5 \mathrm{ppm}$ & $<5 \mathrm{ppm}$ \\
\hline $\mathrm{Pb}$ & & $30 \mathrm{ppm}$ & $<20 \mathrm{ppm}$ \\
\hline $\begin{array}{l}\text { Total heavy } \\
\text { metals }\end{array}$ & & $50 \mathrm{ppm}$ & $<30 \mathrm{ppm}$ \\
\hline
\end{tabular}

* These are typical results obtained for a quality hydroxyapatite coating. The data significantly exceed the minimum regulatory requirements. ed titanium substrate (LHS white area), is shown in Figure 2a, with an SEM image of the surface topography in Figure $2 b$.

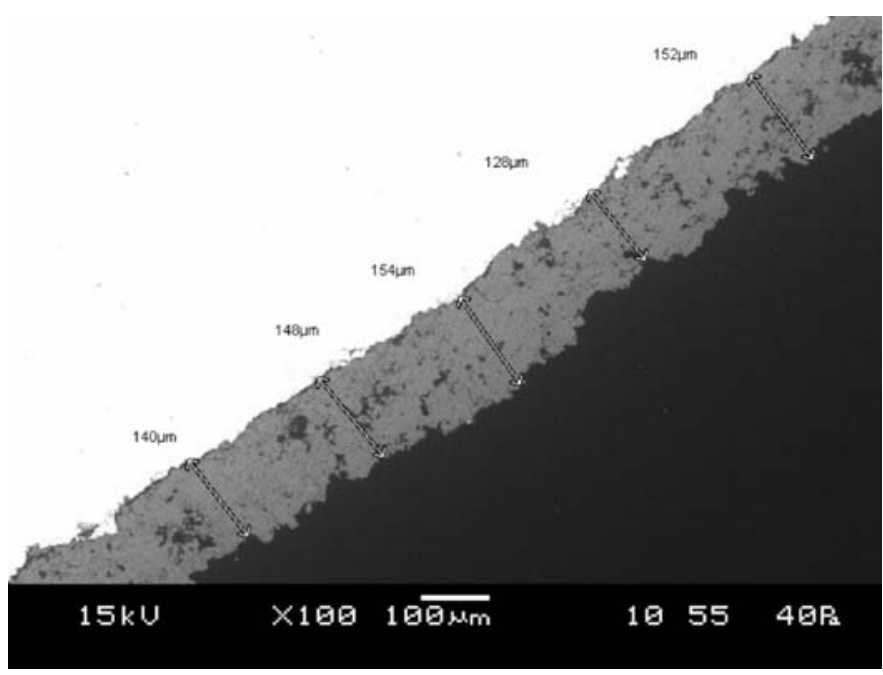

Figure 2a: Cross section of a 130 micron thick Acusure hydroxyapatite coating.

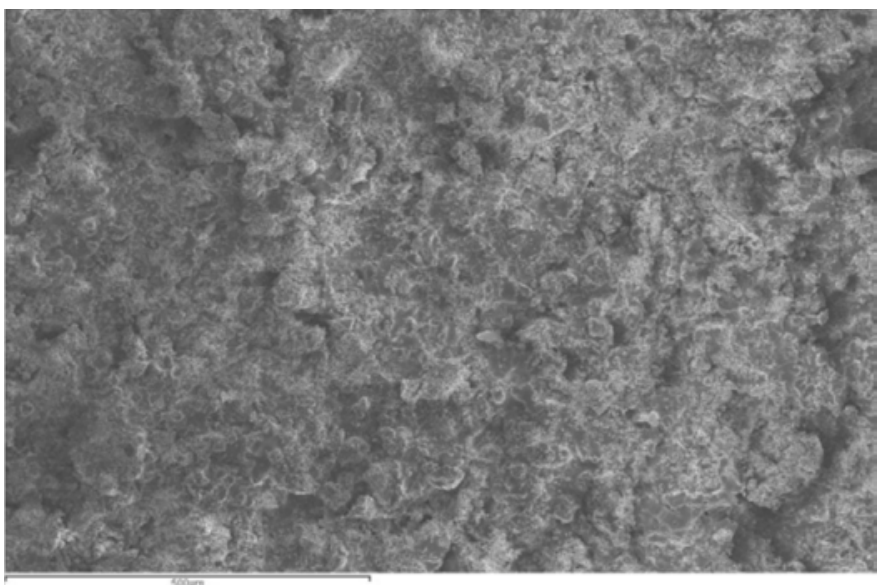

Figure 2b: The surface of an Acusure hydroxyapatite coating.

It is worth noting that the titanium in Figure 1 is deliberately designed as a roughened angular coating, while the hydroxyapatite in Figure 2 is a more homogenous coating.

\section{PRE-CLINICAL TESTING}

In 2002, as part of studies on the validation of plasma spray coatings, a study was commissioned [13] on the effect of coating on early implant osseointegration, using a rabbit model. The study was performed using protocols suggested by two international guidelines:

- "Evaluation of Medical Devices for Biological Hazards: Tests for Local Effects After Implantation." (ISO 10993 part 6)

- Standard Practice for Assessment of Compatibility of Biomaterials for Surgical Implants With Respect to Effect of Materials on Muscle and Bone" (ASTM F981-93) 
In this model, the implants were pins of $3 \mathrm{~mm}$ diameter manufactured from Cobalt/Chrome/Molybdenum alloy. The pins were sequentially shot blasted in order to provide a roughened surface, and coated where appropriate.

The surface coatings investigated within the study were as follows:-

- Uncoated shot blasted pins, used as a control

- Coated with a 100 micron coating of titanium powder

- Bi-coated sequentially with 100 microns of titanium powder and 100 microns of hydroxyapatite

- Bi-coated coated sequentially with 100 microns of cobalt chrome powder and 100 microns of hydroxyapatite

At surgery, each animal received one control pin implanted trans-cortically into the proximal femur and one coated pin distally in the same femur. The animals were sacrificed at either two months or three months and then examined histologically.

Excised femora each containing one implant plug and one control were placed whole into $70 \%$ ethanol. After 48 hours fixation the femora were divided, using a band saw, into proximal and distal sections each containing one implant plug, ensuring that there was at least $2 \mathrm{~mm}$ of bone around each plug. After suitable labelling, the samples were dehydrated through a series of graded alcohols, defatted in acetone, returned to $100 \%$ alcohol prior to infiltration with methyl methacrylate. Samples were polymerised with fresh methyl methacrylate at $55^{\circ} \mathrm{C}$.

The blocks were trimmed using a diamond band saw to expose the implant in a transverse section of the bone. Two 300 micron slices were prepared with the band saw. These slices were then attached to glass slides using hot quartz wax and a compression jig. These sections were then ground to an optical thickness using 30 micron grade aluminium oxide in ethane diol $(10 \% \mathrm{w} / \mathrm{v})$ as an abrasive. Sections were finally stained with Toluidine Blue and mounted with a cover glass and DPX mounting medium.

Specimens were examined visually using transmission bright filed light microscopy using x 20 and $\times 40$ objectives. To verify the findings of these examinations, quantitative histomorphometry was then performed at the implant bone interfaces. This was performed utilising OsteoMeasure ${ }^{\mathrm{TM}}$ software to quantify osteoid surface, osteoid volume and eroded surface.

Representative examples of the visual findings are shown in Figures 3 and 4.

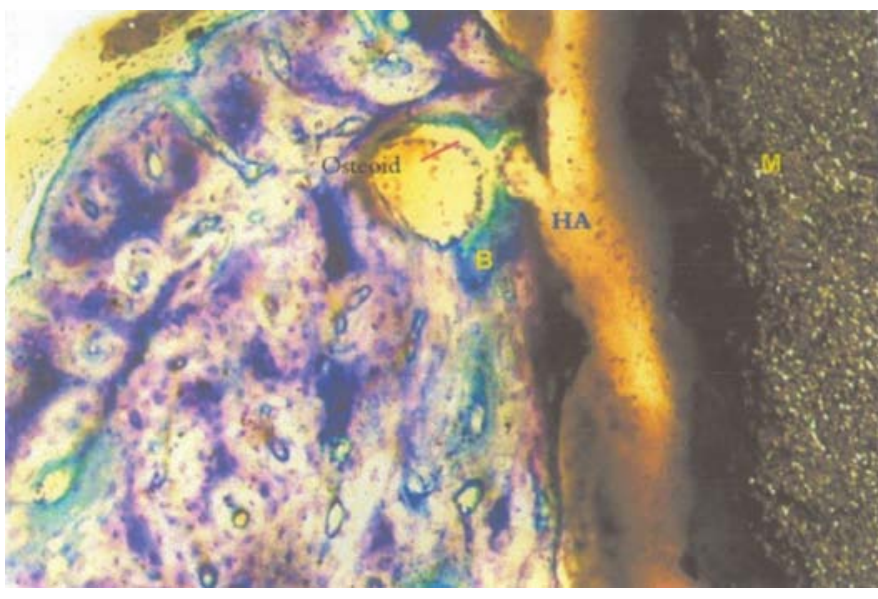

Figure 3: Interface of bone and metal implant $(M)$ with a bi-coating of titanium and hydroxyapatite (HA). Actively forming bone (B) with osteoid seams is clearly visible both at and near the bone implant interface. The bone was stained with Toluidine Blue and was observed with a 20 objective, three months after implantation.

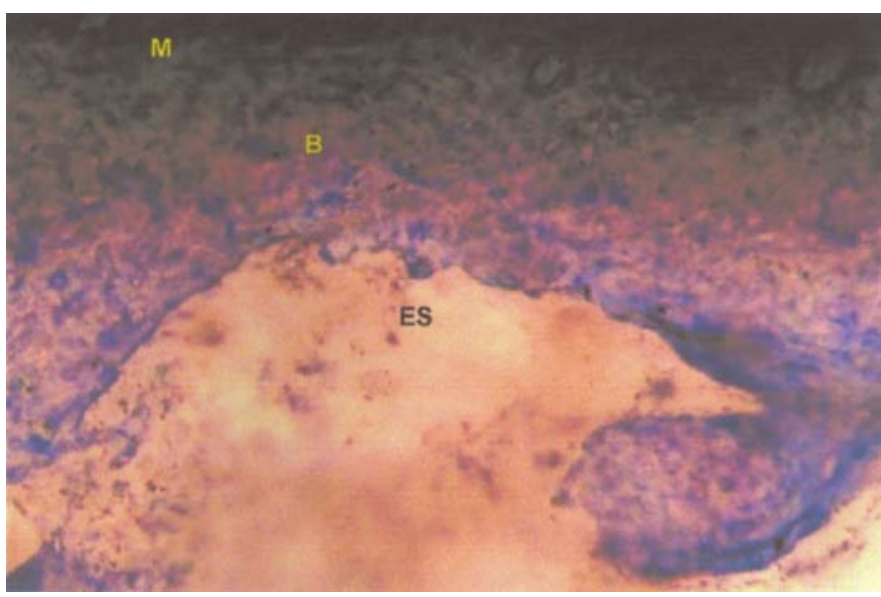

Figure 4: Bone surfaces in the vicinity of the control uncoated metal implant $(M)$ showing very little osteoid. Instead the bone surface is irregular, in some places appearing to have been recently eroded $(E S)$. The bone (B) was stained with Toluidine Blue and viewed with a $x 20$ objective 3 months after insertion of the implant.

The results of the quantitative histomorphometry are shown in Table 3.

Although the number of animals in the study was relatively small, the main reported findings of the study can be summarised as follows:

- The metal coatings or sequential coatings of metal plus hydroxyapatite significantly improved osseointegration and new bone growth compared to uncoated controls.

- There was a suggestion that sequential coatings of metal followed by hydroxyapatite promoted a more rapid normalisation of bone growth at the implant interface than plasma sprayed metal only coatings. This may be important as it would theoretically shorten healing times and allow earlier weight bearing for patients following surgery. 
Table 3. Summary of Quantitative Histomorphometric Analysis

\begin{tabular}{|r|c|c|}
\hline & Osteoid volume \% & Osteoid Surface \% \\
\hline Uncoated 3 months & & \\
\hline Mean & 0.444 & 8.443 \\
\hline Std Error & 0.161 & 1.948 \\
\hline $\mathrm{N}$ & 3 & 3 \\
\hline Coated 2 months & & \\
\hline Mean & 0.748 & 11.278 \\
\hline Std Error & 0.220 & 2.771 \\
\hline $\mathrm{N}$ & 3 & 3 \\
\hline Coated 3 months & & $21.919^{*} \#$ \\
\hline Mean & $2.347^{*} \#$ & 1.022 \\
\hline Std Error & 0.397 & 3 \\
\hline $\mathrm{N}$ & 3 & \\
\hline
\end{tabular}

* Significantly different from uncoated implant at 3 months $P<0.05$

\#Significantly different from coated implant at two months $P<0.05$

\section{Clinical Use of Thermal Plasma Sprayed Coatings}

Over 16 years of clinical use, with over 200,000 implanted devices, there have been no reports of any implant failure which could be attributed to problems related to Accentus Medical's Acusure ${ }^{\circledR}$ coating or adverse bone / tissue reaction. The development of good quality coating processes has resolved challenges from cleaning, entrapment of grit media, oil or polishing compounds. The coatings have been used in primary and revision hip and knee prostheses, shoulder and elbow replacements, tumour replacement implants and pedicle screws. The coatings have been applied to implants manufactured by many different customers and implantations have taken place successfully in every continent of the world.

To illustrate the efficacy of hydroxyapatite coating in a clinical setting, Figure 5 displays Scanning Electron Microscopy of a cross section of a coated device showing formation of viable living bone at the device surface.

The presence of viable new bone, even in such difficult cases as limb salvage surgery, provides good evidence of the biocompatibility and efficacy of thermal plasma sprayed hydroxyapatite coatings.

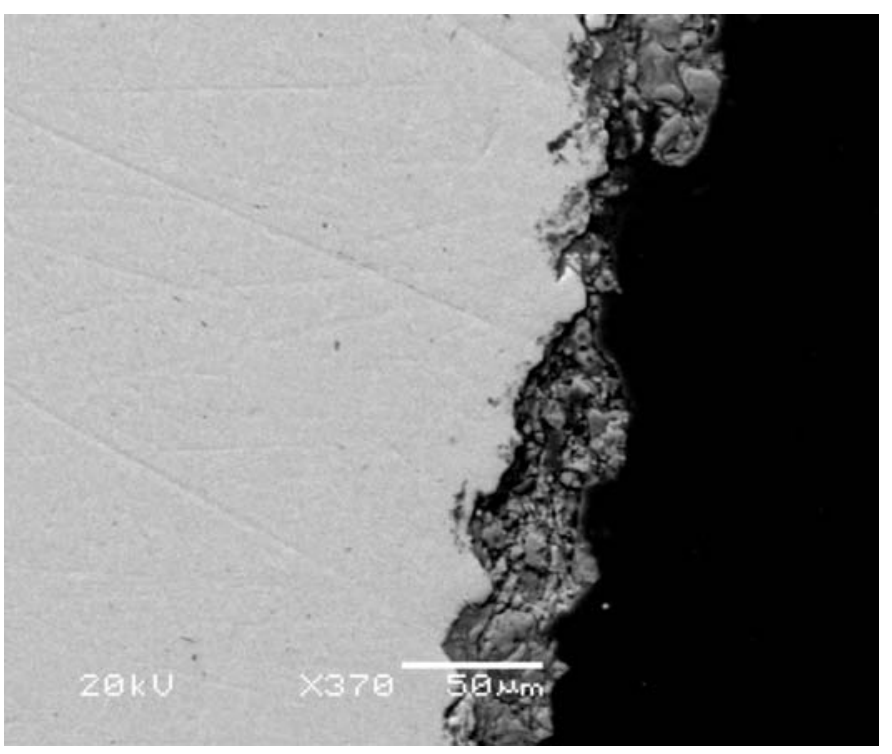

Figure 5: Scanning electron microscope image showing detail of new viable bone formation into a hydroxyapatite coated implant collar manufactured from cobalt chrome alloy. (Image of custom prosthesis explanted from a patient undergoing complex limb salvage surgery).

\section{Summary}

This paper summarises the early history of cementless prostheses and subsequent development, specification, validation, regulatory requirements and clinical performance of thermal plasma spray coatings provided by Accentus Medical. The growth in their use is confirmed by the National Joint Registry of England, Wales and Northern Ireland, which reports that over half of total joint replacements are currently cementless, with good long-term outcomes. Globally, there have been several million implantations of devices incorporating such coatings, with very few reports of issues related to any aspect of the coating itself. Cementless prostheses, with Accentus Medical's Acusure ${ }^{\circledR}$ plasma spray coatings, have good long-term outcomes with over 200,000 implanted devices over a sixteen year history. Many products incorporating this technology have received PMA or 510(K) approvals for the USA market and many have also been awarded an (A) rating by the Orthopaedic Data Evaluation Panel (ODEP) [15]. Thermal plasma spray coatings are a tried and tested technology that have brought benefits to millions of patients around the world for the last thirty years.

\section{Disclosure}

The authors declare that there is no conflict of interest regarding the publication of this paper. For full disclosures refer to last page of this journal. 


\section{References:}

1. Cement Disease. Jones L.C., Hungerford D.S. - Clin Orthop Related Res 1987 (225) 192-206.

2. 13 year follow up study of Harris Galante type hip prostheses in total hip arthroplasty. - Inoue S. Et al -J Orthop Sci 2000;5 (6) : 561-6.

3. Clinical results of the Midstem PCA cementless femoral stem. Knight J.L. et al J Arthroplasty 1998;13 (5): :535 - 545.

4. Outcome of the cementless Taperloc Stem. Mclaughin J.R. Acta Orthop - October $2011 ; 82(5): 633-634$.

5. National Joint Registry of England, Wales and Northern Ireland Annual report 2014.

6. Australian Orthopaedic Joint Registry annual report 2014.

7. HA ceramic coated femoral stems in young patients Sing S. Et al -JBJS (Br) 2004;86-B:1118 -23.

8. Twenty year results of the cementless Corail stem Vidalain J. Et al Int Orthop 2011,35 (2): 189-194.

9. Cementless HA coated total knee replacement. Results at 10 to 12 years. Hutchinson J.R. et al. JBJS (Br) 2006; 86-B Ao supp186.
10. 'Guidance for Industry on the Testing of Metallic Plasma Sprayed Coatings on Orthopaedic Implants to Support Reconsideration of Postmarket Surveillance Requirements' Issued February 2, 2000 by U.S. Department of Health and Human Services, Food and Drug Administration, Centre for Devices and Radiological Health (CDRH).

11. '510(k) Information Needed for hydroxyapatite Coated Orthopaedic Implants' March 101995 (revised 2/20/97), issued by the U.S. Department of Health and Human Services, Food and Drug Administration, Centre for Devices and Radiological Health (CDRH).

12. Global Harmonisation Task Force, GHTF/SG3/N99-10:2004, "Quality Management Systems - Process Validation Guidance".

13. "Assessment of early osseointegration as a function of coating material in rabbit femoral implants." Report BR/JF/06 prepared by AEA Technology (now Accentus Medical Ltd) - August 2002.

14. Late effects of radiation on mature and growing bone. Ramuz O. et al. Cancer Radiother 1997; 1 (6): 801-809.

15. Orthopaedic Data Evaluation Panel - www.ODEP.org.uk.

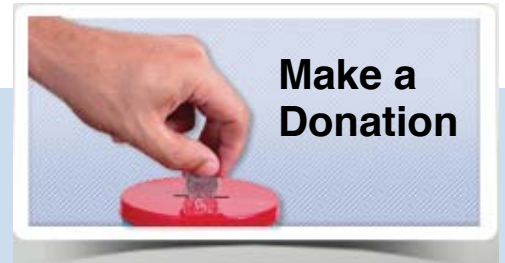

\section{Play a Role in Our Ground Breaking Research}

P

erhaps you were a patient and you were able to

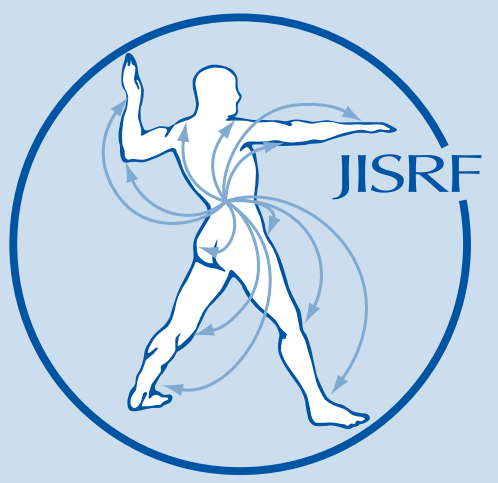
regain an important part of your life. Or, perhaps you are simply someone interested in medical research and seeking a new way to participate. Whatever the case, your generosity in helping to fund research is critical to our success - and much appreciated.

The Joint Implant Surgery \& Research Foundation is a not-for-profit 501(c)(3) corporation. Your contributions enable scientific discoveries that will help future patients. Contributions over the years from people like you have helped to shape orthopaedics today.

\section{Contributions}

Donations of any amount will immediately be put to use to fund ongoing and future orthopaedic research projects.

\section{How to Give}

- Your gift of cash, securities or other negotiable assets is immediately put to use in our research.

- Your contributions are fully tax deductible as specified under Section 501(c)(3) regulations.

For more information please visit our website at www.jisrf.org or contact us at:

\section{Joint Implant Surgery} \& Research Foundation 46 Chagrin Shopping Plaza, \#117 Chagrin Falls, OH 44022 440.785.9154 